Article

\title{
A Naturally Occurring Defective DNA Satellite Associated with a Monopartite Begomovirus: Evidence for Recombination between Alphasatellite and Betasatellite
}

\author{
Changjun Huang, Yan Xie, Liling Zhao, He Ren and Zhenghe Li *
}

Key Laboratory of Molecular Biology of Crop Pathogens and Insects of MOA, Institute of Biotechnology, Zhejiang University, Hangzhou, 310058, China;

E-Mails: cjhuang@zju.edu.cn (C.H.); xiey@zju.edu.cn (Y.X.); zhaolilingyunnan@163.com (L.Z.); renhecau@163.com (H.R.)

* Author to whom correspondence should be addressed; E-Mail: lizh@zju.edu.cn (Z.L.);

Tel.: +86-571-8898-2387; Fax: +86-571-8697-1498.

Received: 2 August 2013; in revised form: 1 September 2013 / Accepted: 2 September 2013 /

Published: 6 September 2013

\begin{abstract}
Monopartite begomoviruses and their associated satellites form unique disease complexes that have emerged as a serious threat to agriculture worldwide. It is well known that frequent recombination contributes to the diversification and evolution of geminiviruses. In this study, we identified a novel defective satellite molecule (RecSat) in association with Tobacco leaf curl Yunnan virus (TbLCYNV) in a naturally infected tobacco plant. Sequence analysis showed that Recsat comprises 754 nucleotides in size and is a chimera involving alphasatellite and betasatellite sequences, containing both betasatellite-conserved region and alphasatellite stem-loop structure. Recombination analysis revealed that RecSat has arisen from three independent recombination events likely involving Tomato yellow leaf curl China betasatellite, Ageratum yellow vein China betasatellite and Tobacco curly shoot alphasatellite. Co-inoculation of RecSat with TbLCYNV induced symptoms indistinguishable from those induced by TbLCYNV alone in Nicotiana benthamiana. Southern blot hybridization showed that RecSat could be trans-replicated stably in $N$. benthamiana plants by TbLCYNV, and impaired the accumulation of helper virus and co-inoculated alphasatellite. Our results provide the first evidence for recombination between two distinct types of satellites among geminivirus complex and highlight recombination as a driving force for geminivirus evolution.
\end{abstract}


Keywords: begomovirus; recombination; alphasatellite; betasatellite; defective DNA satellite

\section{Introduction}

Geminiviruses are a group of plant viruses characterized by their geminate shape particles and circular single-stranded DNA (ssDNA) genomes. Begomovirus, the largest and the most economically important genus of the Geminiviridae family, encompasses viruses that are exclusively transmitted by the whitefly Bemisia tabaci and infect only dicotyledonous plants [1]. Over the last 20 years or so, begomoviruses have emerged as serious constraints to the cultivation of a variety of crops in tropical and subtropical regions worldwide [2,3]. Begomoviruses have genomes consisting of either one or two ssDNA components. The two components of bipartite begomoviruses are designated as DNA-A and DNA-B. In contrast, the monopartite begomoviruses lack the component equivalent to DNA-B, with all viral functions encoded by a single component homologous to DNA-A [4].

To date, two main types of DNA satellites associated with begomoviruses have been described: betasatellites and alphasatellites. Betasatellites, previously referred to as DNA $\beta$, are satellite molecules associated with monopartite begomoviruses and approximately half the size of the helper virus genome ( 1,360 nucleotides in length). Betasatellites modulate disease symptom in most begomovirus/betasatellite complexes and depend on their helper begomoviruses for replication, spreading in plant tissues, encapsidation and insect transmission. The only gene product encoded by betasatellite, $\beta \mathrm{C} 1$, plays an important role in the function of betasatellite. $\beta \mathrm{C} 1$ is a symptom determinant, a suppressor of both transcriptional (TGS) and post-transcriptional gene silencing (PTGS), and can repress plant defenses [5-10]. Alphasatellites, formerly known as DNA 1, are also approximately half the size of begomoviral genomes ( 1,375 nts) and show a conserved genome organization consisting of a single open reading frame (ORF) coding for a replication initiator protein (Rep) [11,12]. Initially, alphasatellites were found in association with the Old World begomovirus/betasatellite complexes. Recently, some distinct alphasatellites were discovered to be associated with the New World begomoviruses $[13,14]$. Although alphasatellites were discovered almost 15 years ago, very little is known about the function(s) of these molecules in begomovirus or begomovirus/betasatellite pathogenesis. Alphasatellite and betasatellite share negligible sequence similarity except for an Adenine-rich (A-rich) region, which is hypothesized to be a stuffer sequence that serves to fulfill the size constrain imposed by helper virus-mediated movement or encapsidation [15].

Besides the full sized betasatellites and alphasatellites, several defective satellites of approximately $700 \mathrm{nts}$ in length have been identified in begomovirus-infected plants. The first identified subviral agent associated with a geminivirus, referred to as Tomato leaf curl virus satellite (ToLCV-sat), is believed to be a defective betasatellite molecule [15]. ToLCV-sat is a small size (682 nts) molecule without ORF, and is strictly dependent on the helper begomovirus for its replication and encapsidation [15]. Recently, similar begomovirus-associated defective satellites have also been identified in malvaceous plants in Cuba and whiteflies in Florida, indicating that this type of satellite molecule is probably common in nature $[16,17]$. However, it is not known whether this type of 
vestigial betasatellite plays any role in disease cycle. At least for ToLCV-sat, it affects neither genome accumulation nor symptom expression of its helper virus [15].

Here, a small recombinant satellite, referred to as RecSat, was found in association with the monopartite Tobacco leaf curl Yunnan virus (TbLCYNV). RecSat is $754 \mathrm{nts}$ in size and resulted from recombination between an alphasatellite and betasatellites. Agroinoculation-based infectivity assays demonstrated that RecSat depends on the helper begomovirus for trans-replication and could modulate the accumulation levels of co-inoculated TbLCYNV and alphasatellite.

\section{Results and Discussion}

\subsection{Results}

2.1.1. Identification of a Defective Betasatellite Molecule in Association with TbLCYNV-Infected Tobacco Plant

Ten tobacco samples showing characteristic geminivirus-like symptoms were collected from Yunnan, China. Total DNA preparations extracted from these samples were subjected to rolling circle amplification (RCA) followed by conventional PCR. Amplification with the primer pair PA/PB, a begomovirus DNA-A degenerate primer pair [18], yielded a 500-base pair (bp) product from each sample (data not shown), confirming the begomovirus infection of these samples. To screen for the presence of betasatellite, universal primer pair beta01/beta02 [19] was used in PCR amplification. A fragment of $\sim 1.3$ kilobase $(\mathrm{kb})$ was obtained from eight of these 10 samples (Figure 1A). Interestingly, a $\sim 0.75 \mathrm{~kb}$ rather than $1.3 \mathrm{~kb}$ band was detected from the sample YN60 (Figure 1A). This $0.75 \mathrm{~kb}$ fragment was cloned and sequenced, and named RecSat. The presence of alphasatellite was also confirmed by PCR in YN60 sample when amplified with the universal alphasatellite-specific primer pair UN101/UN102 [20]. The amplified DNA-A and alphasatellite DNA fragments from YN60 were cloned and sequenced. Alignment of the determined nucleotide sequence showed that YN60 DNA-A and alphasatellite share the highest nucleotide similarities ( 94\%) with TbLCYNV and Tobacco curly shoot alphasatellite (TbCSA) ( 98\%), respectively. All attempts to detect a possible DNA-B component by PCR with DNA-B degenerate primer pairs PCRc1/PBL1v2040 and CR01/CR02 as described [21] from the sample YN60 were unsuccessful, supporting the previous finding that TbLCYNV is a monopartite begomovirus [22].

\subsubsection{Molecular Characterization of RecSat}

Based on the determined RecSat sequence, another pair of abutting primer (RecSatSF and RecSatSR) were designed for PCR amplification, which yielded a specific product of expected size and thus confirmed the circular nature of RecSat (data not shown). Sequence analysis showed that RecSat genome contains 754 nts (GenBank Accession No. KF042891) and has no intact ORF or the A-rich region, the latter of which is a hallmark of betasatellites and alphasatellites. However, RecSat contains several features of begomovirus-associated betasatellites, including a stem-loop with the conserved nonanucleotide TAATATTAC, and iteron-like motifs located just upstream of the stem-loop structure (Figure 1B, top panel). Interestingly, another predicted hairpin structure with a 
loop containing another nonanucleotide TAGTATTAC, which is identical to those of nanoviruses and alphasatellites, was found in the complementary strand of the RecSat. Franking the nonanucleotide of RecSat are some cis-elements and regulatory motifs found in the putative promoter sequence of the Rep gene of alphasatellite, including TATA-box, DOF motifs (AAAAG), and the 5' truncated Rep coding sequence (Figure 1B, bottom panel). The apparent chimeric nature of RecSat suggests that this molecule has been generated as a result of recombination between alphasatellite and betasatellite.

Figure 1. Sequence analysis of RecSat. (A) Agarose gel electrophoresis of PCR-amplified products of ten tobacco samples with beta01 and beta02 primers. The DNA products electrophoresed in lane 1-10 were amplified from sample No. YN51-60, respectively. M denotes size marker lane and $\mathrm{NC}$ denotes the healthy control lane. (B) Schematic representation of the modular organization of the RecSat betasatellite-like SCR (upper panel) and alphasatellite-like replication origin (lower panel). The arrows represent the position and orientation of the putative iterons. Repeated sequence motifs in RecSat are shown in the box. The triangles with a number on the top indicate the number of spacing nucleotides between elements.

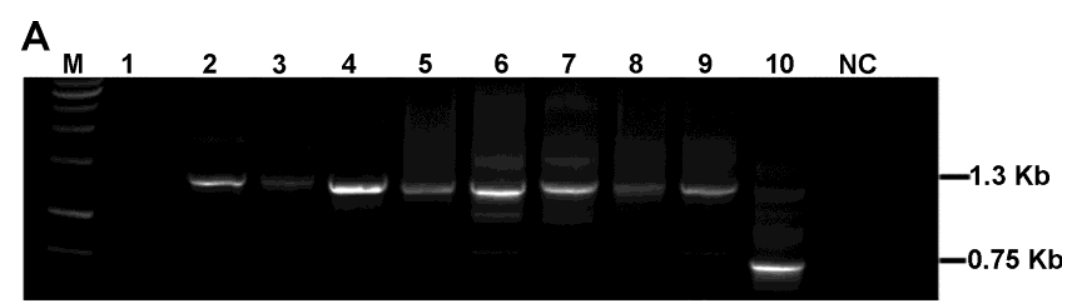

B
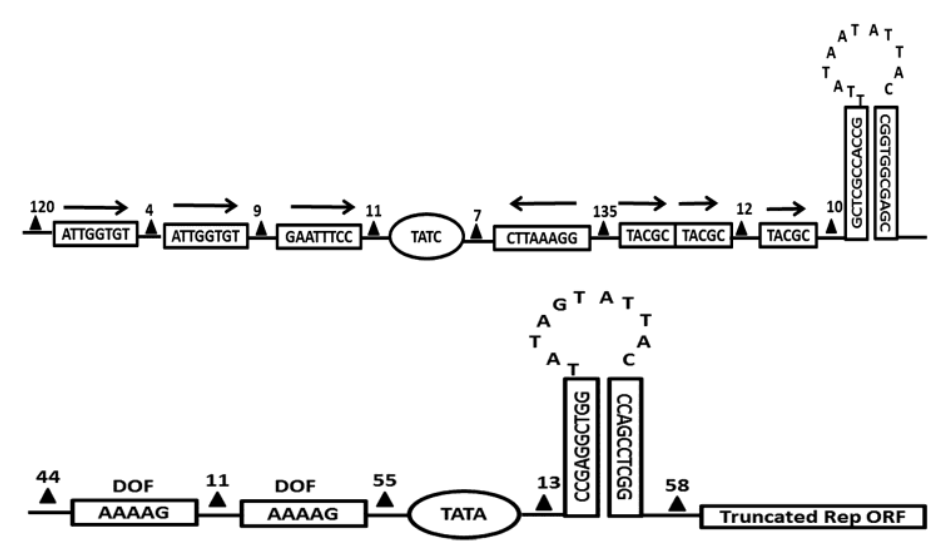

\subsubsection{Recombination Analysis of RecSat}

Next, we aligned the RecSat, betasatellite and alphasatellite genome sequences obtained from public sequence databases using ClustalX software (default settings) [23], followed by recombination analysis using the methods (RDP, GENECONV, Bootscan, MaxChi, Chimaera, SiScan, and 3Seq) included in the RDP3 package with default settings. Three potentially significant recombination events were identified with a high degree of confidence by seven recombination detection methods implemented in RDP3 (Figure 2A). The first potential recombinant fragment of $357 \mathrm{nt}$ in size located from nt 421-21 in RecSat (The third adenosine residue within the nonanucleotide TAATTAC derived from betasatellite was defined as the No. 1 nucleotide position). This fragment contains the conserved 
common region (SCR) of betasatellite, which was likely derived from Tomato yellow leaf curl China betasatellite (TYLCCNB) isolate Y263 (TYLCCNB-Y263). The second potential recombinant region 383-422 shares the highest similarity with Ageratum yellow vein China betasatellite (AYVCNB) isolate G69 (AYVCNB-G69). The third potential recombinant fragment (region 22-384), which contains the alphasatellite conserved stem loop structure and the 5' end of the Rep ORF, can be traced back to TbCSA isolate Y290 (TbCSA-Y290)-like ancestors.

Figure 2. Recombination analyses illustrating the positions of recombination events 1,2 and 3. The nucleotide position 1 of RecSat has been defined as the A residue immediately downstream of the putative nick site within the SCR (TAATTAC). (A) Schematic representation of the three recombination events detected by RDP3 package. Seven different methods available in RDP3 were applied and the obtained confidence values were showed for each recombination event. (B-D) Neighbor-joining trees were constructed using the RecSat region nt 413-21(B), region nt 383-422 (C) and region nt 22-384 (D), and the most representative betasatellite and alphasatellite isolates from Yunnan, China. Accession numbers are shown in figure and the names of the satellites are as follows: AYVB, Ageratum yellow vein betasatellite; AYVCNB, Ageratum yellow vein China betasatellite; TYLCB, Tomato yellow leaf curl betasatellite; TYLCCNB, Tomato yellow leaf curl China betasatellite; TYLCA, Tomato yellow leaf curl alphasatellite; TbCSA, Tobacco curly shoot alphasatellite; TbLCYNA, Tobacco yellow leaf curl Yunnan alphasatellite. (E) Schematic representation of the TYLCCNB-Y263, AYVCNB-G69, TbCSA-Y290 and RecSat. The main genome features such as ORFs, satellite conserved region (SCR) and A-rich region are shown. Sequence regions implicated in recombination event 1, 2 and 3 are displayed in red, dark red and blue, respectively. (F) Comparisons of nucleotide sequences of RecSat with TYLCCNB, AYVCNB and TbCSA around junctions. Residues shared by parental sequences at the junction points are shown in green box.

\begin{tabular}{|c|c|c|c|c|c|c|c|c|c|c|c|}
\hline \multirow{2}{*}{$\begin{array}{c}\text { Event } \\
\text { no. }\end{array}$} & \multicolumn{2}{|c|}{$\begin{array}{c}\text { Breakpoint } \\
\text { position }\end{array}$} & \multirow{2}{*}{$\begin{array}{l}\text { Minor } \\
\text { parent }\end{array}$} & \multirow{2}{*}{$\begin{array}{l}\text { Major } \\
\text { parent }\end{array}$} & \multicolumn{7}{|c|}{ Detection method } \\
\hline & Begin & End & & & RDP & GENECONV & Bootscan & Maxchi & Chimaera & SiScan & 3 Seq \\
\hline 1 & 413 & 21 & TYLCCNB-Y92 & TYLCCNB-Y263 & $1.225 \times 10^{-12}$ & $5.641 \times 10^{-26}$ & $2.461 \times 10^{-18}$ & $1.099 \times 10^{-20}$ & $1.152 \times 10^{-\mathrm{ll}}$ & $1.382 \times 10^{-34}$ & $7.37 \times 10^{-06}$ \\
\hline 2 & 383 & 422 & AYVCNB-G86 & AYVCNB-G69 & $3.106 \times 10^{-05}$ & $5.511 \times 10^{-12}$ & - & $7.149 \times 10^{-06}$ & $1.616 \times 10^{-02}$ & - & $2.518 \times 10^{-03}$ \\
\hline 3 & 22 & 384 & Unknown & TbCSA-Y290 & $8.756 \times 10^{-08}$ & $1.956 \times 10^{-16}$ & $6.489 \times 10^{-14}$ & $2.682 \times 10^{-16}$ & $7.659 \times 10^{-08}$ & $6.819 \times 10^{-20}$ & $5.795 \times 10^{-5}$ \\
\hline
\end{tabular}

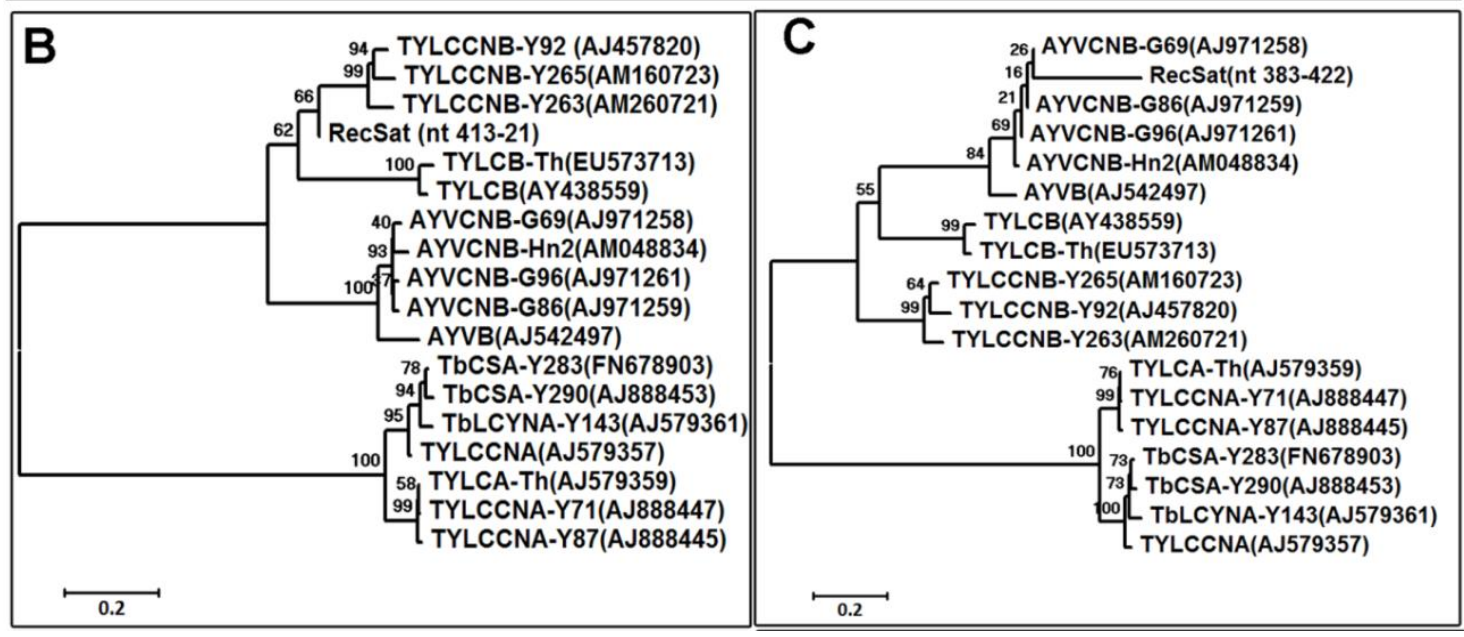


Figure 2. Cont.

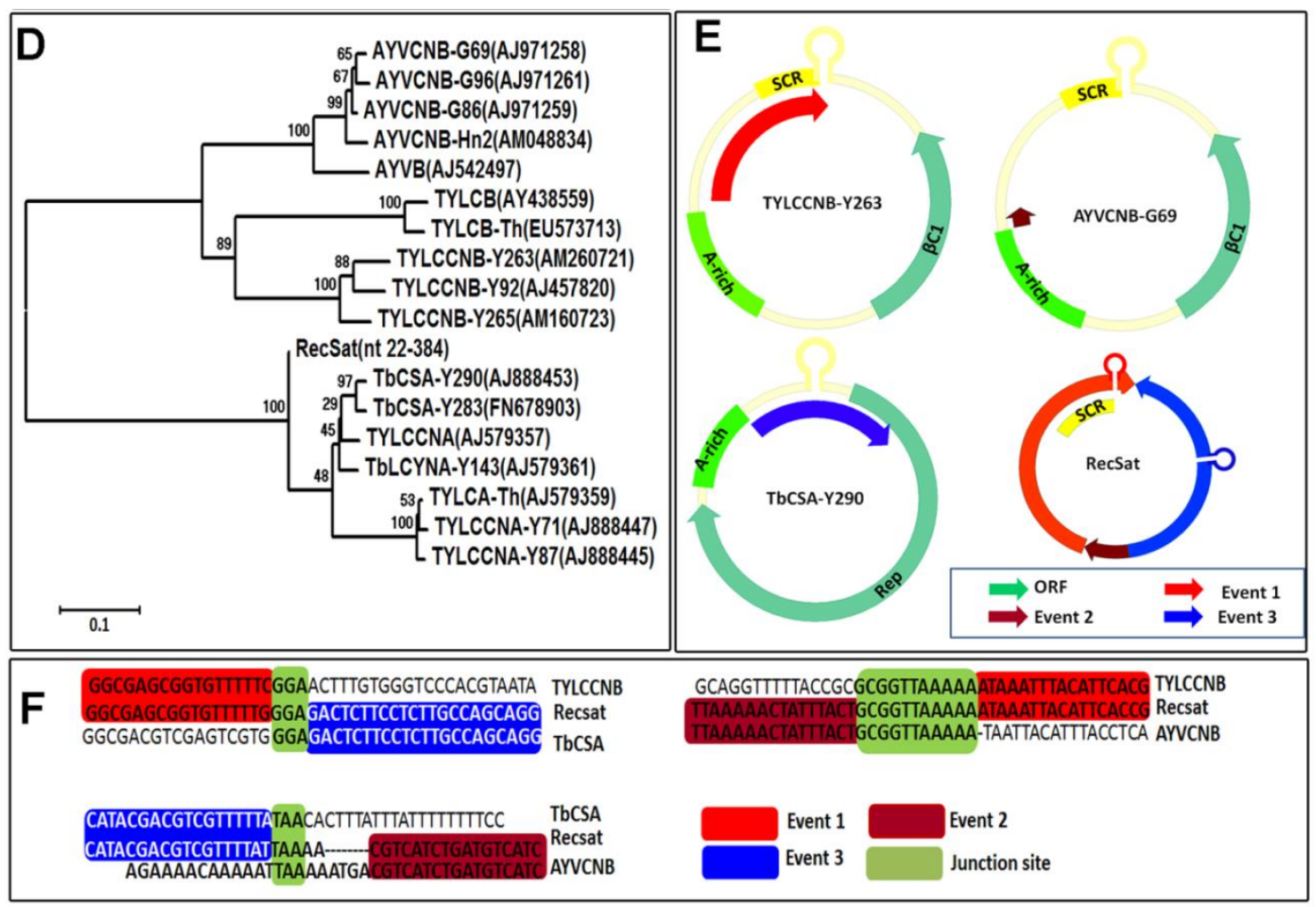

To confirm the phylogenetic relationship, the three relevant regions, divided on the basis of recombination events (event 1: nt 421 to 21, event 2: nt 383 to 422 and event 3: nt 22 to 384), were separately analyzed on neighbor-joining trees with representative betasatellite and alphasatellite isolates. The recombinant RecSat clustered closely with TYLCCNB and AYVCNB sequences, based on event 1 (Figure 2B) and event 2 regions (Figure 2C), respectively, whereas event 3 region clearly clustered with TbCSA sequences (Figure 2D), confirming the occurrence of a triple recombination event.

Further inspection of the recombination breakpoints identified some common sequences between the parental sequences for each recombination event. For example, GGA and TTA were found at the junction site of TYLCCNB/TbCSA, and of TbCSA/AYVCNB, respectively, while a longer stretch of sequence GCGGTTAAAAA was found at the TYLCCNB/AYVCNB junction site (Figure 2F). This suggested that all three recombination events were facilitated by the homologous nucleotide residues of their parental sequences.

\subsubsection{Transreplication of RecSat by TbLCYNV in Nicotiana benthamiana}

To investigate the pathogenicity of this natural recombinant molecule, an infectious clone of RecSat was constructed and agroinoculated into $N$. benthamiana either alone or together with TbLCYNV or TbLCYNV/TbCSA. The infectious clone containing tandem-repeat of the RecSat failed to produce any symptom when agro-inoculated into $N$. benthamiana alone (Figure 3A). Co-inoculation of RecSat and TbLCYNV produced comparable symptom (e.g., severe upward curling of leaves, vein thickening or stunt symptoms) as those induced by TbLCYNV alone in $N$. benthamiana (Figure 3A). In addition, TbLCYNV, TbCSA and RecSat together infected in N. benthamiana resulted in mild leaf-curling, vein 
thickening or stunt symptoms, which are similar to the symptoms observed in the TbLCYNV and TbCSA co-infected $N$. benthamiana plants. These results demonstrated that RecSat had no significant effect on the symptom development of TbLCYNV.

Figure 3. Effect of RecSat on the symptom development and DNA accumulation of helper virus. (A) Symptoms induced by TbLCYNV when co-inoculated with either RecSat or TbCSA/RecSat in Nicotiana benthamiana at 15 dpi. (B) Southern blot analysis of TYLCCNB, TbCSA and RecSat accumulation in N. bethamiana plants with specific probes.
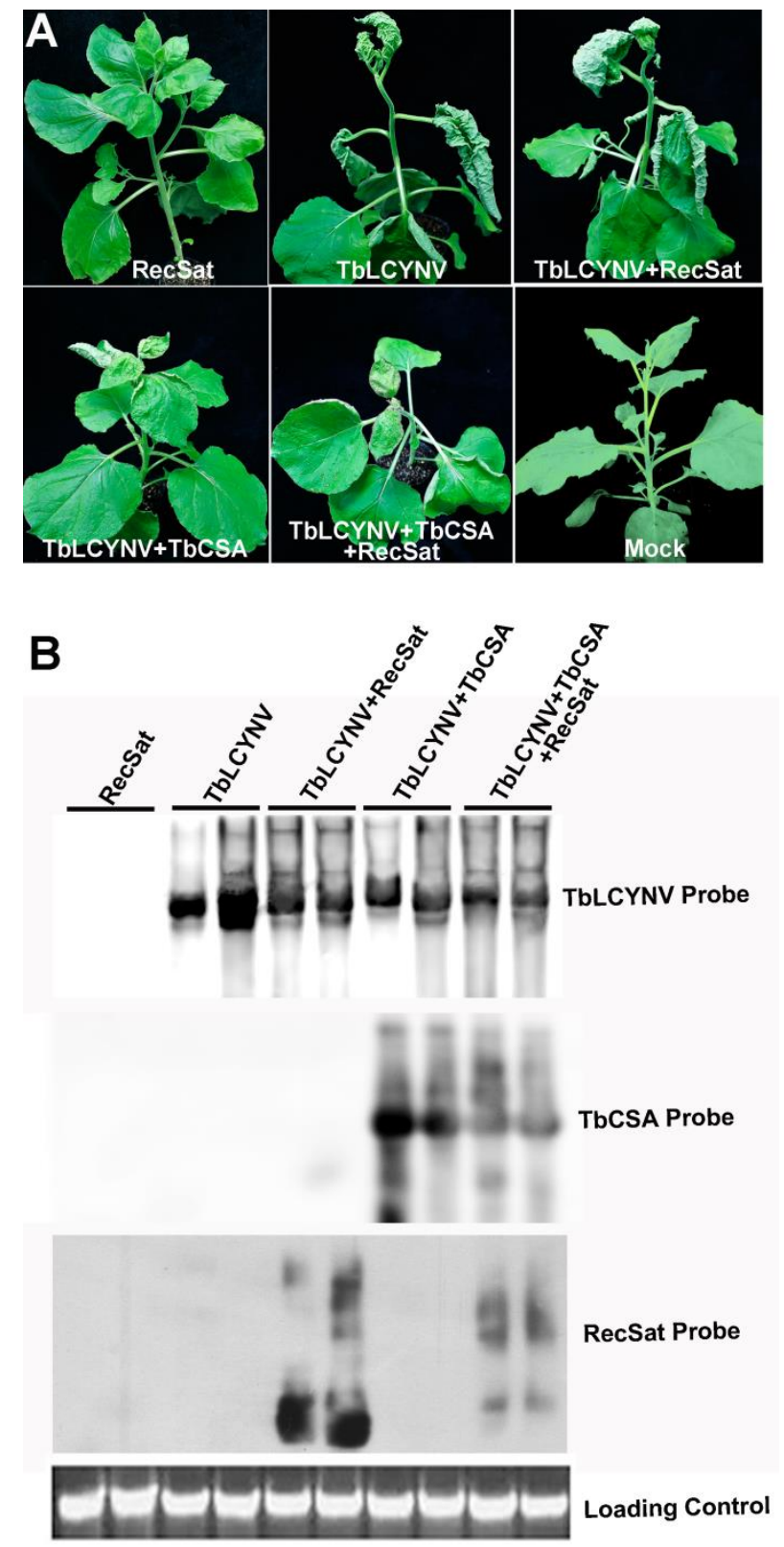

Southern blot analysis showed that RecSat alone could not systemically spread and accumulate in $N$. benthamiana. When co-inoculated with TbLCYNV, high levels of the RecSat were detected in systemically infected tissue, indicating efficient trans-replication and systemic movement mediated by the helper virus. Comparison of TbLCYNV genomic DNA levels showed that RecSat interfered with helper virus accumulation in co-inoculated plants tissues. When RecSat was co-inoculated with 
TbLCYNV and TbCSA, attenuations of both TbLCYNV and TbCSA DNA accumulation were also observed. Therefore, these results demonstrated that RecSat could be trans-replicated and stably maintained by TbLCYNV in plants and has a negative effect on the accumulation of TbLCYNV and TbCSA.

\subsection{Discussion}

It is well recognized that genetic recombination plays a major role in the diversification and evolution of geminiviruses [24-26]. Recombination has been documented to occur between geminivirus, between betasatellites, alphasatellites and between helper viruses and betasatellites [24,27-30]. In the present work, we characterized a novel chimeric molecule (RecSat) resulting from recombination between betasatellite and alphasatellite. Using computer program-based recombination analysis, we provided strong evidence that the RecSat genome was generated through three independent recombination events. Inspection of the junction site identified 3-10 common nucleotide residues (e.g., GGA, TAA and GCGGTTAAAAA) shared by parental molecules involved in the recombination events. Such short stretches of common sequences have also been found at the junction site of progenitor geminiviral sequences implicated in other recombination events [28,29]. These results suggest that sequence homology may play an important role in recombination within geminiviruses and their associated satellites.

The identified "parents" of the recombinant RecSat include TYLCCNB, AYVCNB and TbCSA, all of which are frequently found in association with tobacco-infecting begomoviruses from Yunnan $[11,31,32]$. Since these "donor" satellites and RecSat have natural hosts in common and prevail in the same geographic location, it is probable that the recombination event may have occurred in co-infected tobacco plants. Alternatively, recombination might have occurred in weeds followed by insect transmission to tobacco plants, since it is well known that weeds are intermediate hosts and reservoirs of begomoviruses and act as "melting pots" that yield new viruses/virus strains by recombination due to their frequently harboring multiple viruses [2].

The SCR of betasatellite contains several important cis-elements required for trans-replication, including the Rep binding sites and a conserved stem-loop motif that includes the nick site for the initiation of rolling circle replication [33]. All hitherto characterized defective betasatellite molecules retain the SCR, highlighting the importance of this region in the maintenance of betasatellite [15-17]. As with defective betasatellites, RecSat also contains the conserved betasatellite stem-loop structure and flanking repeated sequence motifs (Figure 1B) that presumably are involved in recruiting virus-encoded Rep to initiate replication. Indeed, RecSat accumulated to high levels in the presence of TbLCYNV. Notably, RecSat accumulates at the expense of helper virus replication (Figure 3B). This is reminiscent of defective interfering DNAs, which also appear to decrease helper virus accumulation [20]. It is worth noting that RecSat contains, besides SCR, an alphasatellite-derived replication origin (Figures $1 \mathrm{~B}$ and $3 \mathrm{E}$ ), and also interferes with the accumulation of the co-inoculated alphasatellite TbSCA. It is tempting to speculate that alphasatellite alone could mediate the replication of RecSat. Further study is needed to determine whether the alphasatellite-derived replication origin is involved in RecSat replication and interference of the accumulation of both helpers and alphasatellites. 
Previous study has showed that only a few isolates of TbLCYNV are associated with betasatellite molecules [21]. Moreover, TbLCYNV alone is able to induce severe symptoms, and co-inoculation with betasatellites does not further intensify the symptoms [22]. Thus, TbLCYNV resembles a true monopartite begomovirus such as ToLCV more than a helper/betasatellite disease complex. The monopartite nature of TbLCYNV may allow defective recombinant molecules as RecSat to be maintained in the absence of functional betasatellite, a scenario reminiscent of ToLCV-sat (15). By contrast, other betasatellite-dependent helper viruses, including Tomato yellow leaf curl China virus, Cotton leaf curl Multan virus and Ageratum yellow vein virus would not prevail in the absence of functional betasatellite. Accordingly, all naturally occurring betasatellite recombinants in association with these helper viruses maintain intact $\beta C 1$ gene and are able to induce typical symptoms when co-inoculated with helper viruses [27,28]. Although RecSat appears to have no discernible effect on symptom development in $N$. benthamiana (Figure 3B), its ability to alter helper virus accumulation suggests a biological role as a defective interfering DNA. Further survey of similar molecules in plants and whiteflies will be performed to evaluate their diversity and potential role in disease maintenance.

\section{Experimental}

\subsection{Sources of Virus Isolates}

Ten leaf samples were collected from naturally infected tobacco plants showing begomovirus-like infection symptoms from Yunnan Province, China during the summer of 2003. Total DNAs were extracted from these samples using a CTAB-based method, and rolling-circle amplification (RCA) was performed using a Templiphi ${ }^{\mathrm{TM}}$ DNA Amplification Kit (GE Healthcare, Piscataway, NJ, USA) as described [34].

\subsection{PCR Amplification and Cloning of RecSat}

Presence of begomovirus in samples was investigated by PCR using RCA product as template and the degenerate primer pair PA/PB as described [34]. In parallel, alphasatellites and betasatellites were analyzed using abutting primers UN101 (5'-AAGCTTGCGACTATTGTATGAAAGAGG-3')/UN102 (5'-AAGCTTCGTCTGTCTTACGAGCTCGCTG-3'), and beta01/beta02 [19] specific to alphasatellites and betasatellites, respectively. The PCR products were purified, and cloned using pGEM-T Easy Vector (Promega, Madison, WI, USA) and sequenced using an automated DNA sequencing system (Model 377; Perkin Elmer, Foster City, CA, USA).

\subsection{Sequence Analysis}

Database searches with the RecSat sequences were carried out by NCBI-BLAST program [35]. Pairwise comparisons were conducted with the ClustalX program using default parameters [23] and phylogenetic inference was performed using MEGA 5 by the neighbor-joining method [36]. Recombination analysis was performed using the methods included in the RDP 3.0 package with default settings [37]. To confirm the recombination results, the relevant fragments from betasatellite or alphasatellite were analyzed on neighbor-joining trees using MEGA 5. 


\subsection{Construction of RecSat Infectious Clone}

To construct the RecSat infectious clone, specific primers RecSatSF (5'-ttacGAGCTCCGGGGAGTTTTTGGAGAGA-3', underlined is an existing SalI restriction site) and RecSatBR (5'-ttatGGATCCCGGGGAGTTTTTGGAGAGA-3', underlined is an existing BamHI restriction site) were designed and used to amplify the full length RecSat genome. The $0.75 \mathrm{~kb}$ PCR product was cloned into pGEM-T Easy vector to produce pGEM-1RecSat. After confirmation by sequencing, a BamHI-SalI digested fragment of pGEM-1RecSat was introduced into the binary plant transformation vector pBINPLUS [38] to produce the clone PBINPLUS-1RecSat. Subsequently, using the same strategy, another copy of a full length RecSat genome was amplified with primers RecSatSF and RecSatSR (5'-ttacGAGCTCCGATTAAGGGTCTTCCGGTA-3', underlined is an introduced SalI restriction site) and SalI-digested followed by insertion into the unique SalI restriction site of PBINPLUS-1RecSat to produce clone PBINPLUS-2RecSat, yielding a 2-mer tandem repeat of RecSat. The infectious clones of TbLCYNV (pBINPLUS-Y143) and TbCSA (pBINPLUS-Y35DNA1) were previously constructed in our lab [20,22].

\subsection{Agroinoculation of Plants}

The binary vector carrying TbLCYNV, TbCSA and RecSat constructs were introduced individually into A. tumefaciens strain EHA105 through electroporation using the GENE PULSER II electroporation system (Bio-Rad Laboratories, Hercules, CA, USA) as instructed by the manufacturer's manual. The transformed A. tumefaciens cultures were incubated individually in a YEP medium containing spectinomycin $(50 \mathrm{mg} / \mathrm{L})$ and rifampicin $(50 \mathrm{mg} / \mathrm{L})$. The cultures were grown overnight inside a shaker set at $28{ }^{\circ} \mathrm{C}$ and $200 \mathrm{rpm}$. The overnight cultures were pelleted and then resuspended in an induction buffer containing $10 \mathrm{mM} \mathrm{MgCl} 2,10 \mathrm{mM} \mathrm{MES}$, and $150 \mu \mathrm{M}$ acetosyringone. After $3 \mathrm{~h}$ of incubation at room temperature, the Agrobacterium cultures were adjusted to OD600 $=0.8$ to 1.0 , and injected into stem or petioles 4 weeks old $N$. benthamiana plants. For co-inoculation, equal volumes of the separate cultures were mixed prior to inoculation. Inoculated plants were grown in an insect-free chamber and observed daily for the presence of symptoms.

\subsection{Analysis of Virus and Satellite DNA Accumulation}

Nucleic acids were isolated from upper leaves at 30 days post inoculation (dpi) and viral DNA accumulation was assayed by Southern blot hybridization as described [39]. In brief, nucleic acids were fractionated in $1 \% \mathrm{w} / \mathrm{v}$ agarose gel, transferred to Hybond $-\mathrm{N}^{+}$membranes (GE Healthcare) and cross-linked with UV illumination. After alkali denaturation and neutralization, nucleic acid accumulation was detected with a random primed $\left[\alpha-{ }^{32} \mathrm{P}\right] \mathrm{dCTP}-$ labeled probe. For analysis of TbLCYNV, a probe containing AC2 and parts of AC1 gene (approximately $710 \mathrm{bp}$ ) was amplified from pBINPLUS-Y143 with Y143F (5'-AAGTCTGAGGTTGTAAGCGAGTC-3') and Y143R (5'-CCATCAGTATTGTCATAGAGGGAG-3') primers as described previously [15]. For specific analysis of TbCSA, a probe contains partial TbCSA (nt 500-800 of TbCSA) was amplified from pBINPLUS-Y35DNA1 with TbCSAPF (5'-cggaggaagaattccaggcta-3') and TbCSAPR (5'-aactatattgcagataatcct-3') primers. For specific analysis of RecSat, a probe containing betasatellite 
homologous sequence (nt 421-721 of RecSat) was amplified from pBINPLUS-2RecSat with RecSatPF (5'-CATTCACCGCATAGGTAAAT-3') and RecSatPR (5'-GCGAGCTAAGCTCCGGCGT-3') primers.

\section{Conclusions}

In summary, we report for the first time the occurrence of recombination between different types of satellites of geminivirus complex in the field. The recombinant satellite interferes with helper virus accumulation, behaving like a defective interfering DNA. Our study highlights the potential of recombination as a driving force for geminivirus diversification.

\section{Acknowledgments}

We thank the anonymous reviewers for their helpful comments in improving the earlier draft of this paper. This work was supported by grants from the National Natural Science Foundation of China (No. 31000839, No. 31171814 and No. 31100114).

\section{Conflicts of Interest}

The authors declare no conflict of interest.

\section{References and Notes}

1. Harrison, B.; Robinson, D. Natural genomic and antigenic variation in whitefly-transmitted geminiviruses (begomoviruses). Annu. Rev. Phytopathol. 1999, 37, 369-398.

2. Mansoor, S.; Briddon, R.W.; Zafar, Y.; Stanley, J. Geminivirus disease complexes: An emerging threat. Trends Plant Sci. 2003, 8, 128-134.

3. Mansoor, S.; Zafar, Y.; Briddon, R.W. Geminivirus disease complexes: The threat is spreading. Trends Plant Sci. 2006, 11, 209-212.

4. Jeske, H. Geminiviruses. Curr. Top. Microbiol. Immunol. 2009, 331, 185-226.

5. Cui, X.F.; Li, G.X.; Wang, D.W.; Hu, D.W.; Zhou, X.P. A begomovirus DNA beta-encoded protein binds DNA, functions as a suppressor of RNA silencing, and targets the cell nucleus. J. Virol. 2005, 79, 10764-10775.

6. Cui, X.F.; Tao, X.R.; Xie, Y.; Fauquet, C.M.; Zhou, X.P. A DNA beta associated with Tomato yellow leaf curl China Virus is required for symptom induction. J. Virol. 2004, 78, 13966-13974.

7. Eini, O.; Dogra, S.; Selth, L.A.; Dry, I.B.; Randles, J.W.; Rezaian, M.A. Interaction with a host ubiquitin-conjugating enzyme is required for the pathogenicity of a geminiviral DNA beta satellite. Mol. Plant Microbe Interact. 2009, 22, 737-746.

8. Shen, Q.; Liu, Z.; Song, F.; Xie, Q.; Hanley-Bowdoin, L.; Zhou, X. Tomato SiSnRK1 protein interacts with and phosphorylates betaC1, a pathogenesis protein encoded by a geminivirus beta-satellite. Plant Physiol. 2011, 157, 1394-1406.

9. Yang, X.; Xie, Y.; Raja, P.; Li, S.; Wolf, J.N.; Shen, Q.; Bisaro, D.M.; Zhou, X. Suppression of methylation-mediated transcriptional gene silencing by betaC1-SAHH protein interaction during geminivirus-betasatellite infection. PLoS Pathog. 2011, 7, e1002329. 
10. Zhou, X. Advances in understanding begomovirus satellites. Annu. Rev. Phytopathol. 2013, 51, $357-381$.

11. Xie, Y.; Wu, P.; Liu, P.; Gong, H.; Zhou, X. Characterization of alphasatellites associated with monopartite begomovirus/betasatellite complexes in Yunnan, China. Virol. J. 2010, 7, 178.

12. Briddon, R.W.; Bull, S.E.; Amin, I.; Mansoor, S.; Bedford, I.D.; Rishi, N.; Siwatch, S.S.; Zafar, Y.; Abdel-Salam, A.M.; Markham, P.G. Diversity of DNA 1: A satellite-like molecule associated with monopartite begomovirus-DNA beta complexes. Virology 2004, 324, 462-474.

13. Idris, A.M.; Shahid, M.S.; Briddon, R.W.; Khan, A.J.; Zhu, J.K.; Brown, J.K. An unusual alphasatellite associated with monopartite begomoviruses attenuates symptoms and reduces betasatellite accumulation. J. Gen. Virol. 2011, 92, 706-717.

14. Paprotka, T.; Metzler, V.; Jeske, H. The first DNA 1-like alphasatellites in association with New World begomoviruses in natural infections. Virology 2010, 404, 148-157.

15. Dry, I.B.; Krake, L.R.; Rigden, J.E.; Rezaian, M.A. A novel subviral agent associated with a geminivirus: The first report of a DNA satellite. Proc. Natl. Acad. Sci. USA 1997, 94, 7088-7093.

16. Fiallo-Olive, E.; Martinez-Zubiaur, Y.; Moriones, E.; Navas-Castillo, J. A novel class of DNA satellites associated with New World begomoviruses. Virology 2012, 426, 1-6.

17. Ng, T.F.; Duffy, S.; Polston, J.E.; Bixby, E.; Vallad, G.E.; Breitbart, M. Exploring the diversity of plant DNA viruses and their satellites using vector-enabled metagenomics on whiteflies. PLoS One 2011, 6, e19050.

18. Xie, Y.; Zhou, X.; Li, Z.; Zhang, Z.; Li, G. Identification of a novel DNA molecule associated with Tobacco leaf curl virus. Chinese Sci. Bull. 2002, 47, 1273-1276.

19. Zhou, X.P.; Xie, Y.; Peng, Y.; Zhang, Z.K. Malvastrum yellow vein virus, a new Begomovirus species associated with satellite DNA molecule. Chinese Sci. Bull. 2003, 48, 2205-2209.

20. Wu, P.J.; Zhou, X.P. Interaction between a nanovirus-like component and the Tobacco curly shoot virus/satellite complex. Acta Bioch. Bioph. Sin. 2005, 37, 25-31.

21. Cui, X.F.; Xie, Y.; Zhou, X.P. Molecular characterization of DNA $\beta$ molecules associated with Tobacco leaf curl Yunnan Virus. J. Phytopathol. 2004, 152, 647-650.

22. Xie, Y.; Jiang, T.; Zhou, X. Agroinoculation Shows Tobacco leaf curl Yunnan virus is a Monopartite Begomovirus. Eur. J .Plant Pathol. 2006, 115, 369-375.

23. Thompson, J.D.; Gibson, T.J.; Plewniak, F.; Jeanmougin, F.; Higgins, D.G. The CLUSTAL_X windows interface: Flexible strategies for multiple sequence alignment aided by quality analysis tools. Nucleic Acids Res. 1997, 25, 4876-4882.

24. Zhou, X.P.; Liu, Y.L.; Calvert, L.; Munoz, C.; OtimNape, G.W.; Robinson, D.J.; Harrison, B.D. Evidence that DNA-A of a geminivirus associated with severe cassava mosaic disease in Uganda has arisen by interspecific recombination. J. Gen. Virol. 1997, 78, 2101-2111.

25. Rojas, M.R.; Hagen, C.; Lucas, W.J.; Gilbertson, R.L. Exploiting chinks in the plant's armor: Evolution and emergence of geminiviruses. Annu. Rev. Phytopathol. 2005, 43, 361-394.

26. Martin, D.P.; Lefeuvre, P.; Varsani, A.; Hoareau, M.; Semegni, J.Y.; Dijoux, B.; Vincent, C.; Reynaud, B.; Lett, J.M. Complex recombination patterns arising during geminivirus coinfections preserve and demarcate biologically important intra-genome interaction networks. PLoS Pathog. 2011, 7, e1002203. 
27. Saunders, K.; Bedford, I.D.; Stanley, J. Pathogenicity of a natural recombinant associated with Ageratum yellow vein disease: Implications for geminivirus evolution and disease aetiology. Virology 2001, 282, 38-47.

28. Tao, X.; Zhou, X. Pathogenicity of a naturally occurring recombinant DNA satellite associated with tomato yellow leaf curl China virus. J. Gen. Virol. 2008, 89, 306-311.

29. Stanley, J.; Saunders, K.; Pinner, M.S.; Wong, S.M. Novel defective interfering DNAs associated with ageratum yellow vein geminivirus infection of Ageratum conyzoides. Virology 1997, 239, 87-96.

30. Saunders, K.; Stanley, J. A nanovirus-like DNA component associated with yellow vein disease of Ageratum conyzoides: Evidence for interfamilial recombination between plant DNA viruses. Virology 1999, 264, 142-152.

31. Zhou, X.; Xie, Y.; Tao, X.; Zhang, Z.; Li, Z.; Fauquet, C.M. Characterization of DNAbeta associated with begomoviruses in China and evidence for co-evolution with their cognate viral DNA-A. J. Gen. Virol. 2003, 84, 237-247.

32. Jiao, X.; Gong, H.; Liu, X.; Xie, Y.; Zhou, X. Etiology of Ageratum yellow vein diseases in South China. Plant Dis. 2013, in press.

33. Saunders, K.; Briddon, R.W.; Stanley, J. Replication promiscuity of DNA-beta satellites associated with monopartite begomoviruses; deletion mutagenesis of the Ageratum yellow vein virus DNA-beta satellite localizes sequences involved in replication. J. Gen. Virol. 2008, 89, 3165-3172.

34. Zulfiqar, A.; Zhang, J.; Cui, X.; Qian, Y.; Zhou, X.; Xie, Y. A new begomovirus associated with alpha- and betasatellite molecules isolated from Vernonia cinerea in China. Arch Virol. 2012, 157, 189-191.

35. NCBI. Available online: http://blast.ncbi.nlm.nih.gov (accessed on 4 September 2013).

36. Tamura, K.; Dudley, J.; Nei, M.; Kumar, S. MEGA4: Molecular Evolutionary Genetics Analysis (MEGA) software version 4.0. Mol. Biol. Evol. 2007, 24, 1596-1599.

37. Martin, D.P.; Lemey, P.; Lott, M.; Moulton, V.; Posada, D.; Lefeuvre, P. RDP3: A flexible and fast computer program for analyzing recombination. Bioinformatics 2010, 26, 2462-2463.

38. van Engelen, F.A.; Molthoff, J.W.; Conner, A.J.; Nap, J.P.; Pereira, A.; Stiekema, W.J. pBINPLUS: An improved plant transformation vector based on pBIN19. Transgenic Res. 1995, 4, 288-290.

39. Huang, C.J.; Xie, Y.; Zhou, X.P. Efficient virus-induced gene silencing in plants using a modified geminivirus DNA1 component. Plant Biotechnol. J. 2009, 7, 254-265.

(C) 2013 by the authors; licensee MDPI, Basel, Switzerland. This article is an open access article distributed under the terms and conditions of the Creative Commons Attribution license (http://creativecommons.org/licenses/by/3.0/). 